\title{
3D segmented model of head for modelling electrical activity of brain
}

Egill A Friðgeirsson (1,2), Paolo Gargiulo (1,2), Ceon Ramon (2,3), Jens Haueisen (4)

(1) Department of Development CE and IT, Landspitali University Hospital; (2) Department of Biomedical Engineering, University of Reykjavik, Iceland; (3) Department of Electrical Engineering, University of Washington, United States; (4) Institute of Biomedical Engineering and Informatics, Technical University Ilmenau, Germany

\begin{abstract}
Computer simulation and modelling of the human body and its behaviour are very useful tools in situations where it is either too risky to perform an invasive procedure or too costly for in vivo experiments or simply impossible for ethical reasons. In this paper we describe a method to model the electrical behaviour of human brain from segmented MR images. The aim of the work is to use these models to predict the electrical activity of human brain under normal and pathological conditions. The image processing software package MIMICS is used for 3D volume segmentation of MR images. These models have detailed 3D representation of major tissue surfaces within the head, with over 12 different tissues segmented. In addition, computational tools in Matlab were developed for calculating normal vectors on the brain surface and for associating this information to the equivalent electrical dipole sources as an input into the model.
\end{abstract}

Key Words: EEG modeling, finite element, realistic head model, segmentation

European Journal Translational Myology - Basic Applied Myology 2012; 22 (1\&2):57-60

The relationship between neuronal sources and the recorded scalp Electroencephalographs (EEG's) has for a long time been of interest [1]. This relationship is not simple and relies on different coupled non-linear physical mechanisms. The electrical effects of these mechanisms then distribute from their sources through inhomogeneous media with various electrical properties. Forward EEG modelling is a discipline which uses numerical techniques such as finite element modelling (FEM) to study the relationship between electric sources in the brain and the resulting electrical potentials at the scalp [4]. Sources in the form of current dipoles are placed in the brain and then the FEM equations are solved for the resulting potential at the scalp. For accurate forward EEG modelling detailed segmentation of tissues is needed, especially between the electrical source and scalp. In former studies the model complexity, or the number of tissue types, has been shown to affect the results [10]. These have emphasized the role of accurate segmentation of the cerebrospinal fluid (CSF) and bone [9]. The kind of 3D segmentation used here with the software platform MIMICS (Materialize Inc, USA) has previously been applied to monitor the quadriceps femoris muscle in paraplegic patients undergoing electrical stimulation. $[2,3]$. In this work we develop high $(0.5$ to $1.0 \mathrm{~mm})$ resolution human head models from segmented MR images. These models have a detailed 3D representation of major tissue surfaces with over 12 tissue types defined. Brain geometry is then used to locate the position and orientation of the cortical neurons to use as sources in the models. The cortical pyramidal neurons are considered the main contributors to the EEG signal [7]. These models will be used for modelling the electrical activity of brain under normal and pathological conditions and identifying the contribution of different brain areas on the EEG signal.

\section{Materials and Methods}

\subsection{Data set}

The dataset consists of $\mathrm{T} 1$ weighted magnetic resonance (MR) images acquired at the University Hospital in Jena Germany. The data was collected with a 3 tesla Siemens MR scanner and consist of one dataset of a 55 year old female subject. A T1 weighted scan was chosen to maximize the contrast between the 

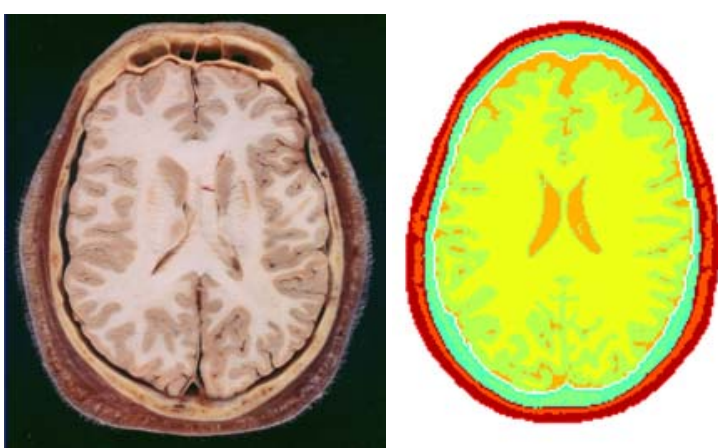

Fig 1 Segmentation results showing different tissue surfaces (right) and an anatomical reference (right). The tissues are represented by different colors.

gray and white matter. The dataset consists of 192 sagittal slices with 256 X 256 pixels each. Each pixel is represented by a 12 bit gray value. In total there are approximately 12.6 million voxels of $1 \times 1 \times 1 \mathrm{~mm}$ size.

\subsection{Segmentation of the head}

A detailed 3D representation of major tissues within the head was developed including white and gray matter, cerebellum, CSF, cortical and trabecular bone, dura layer, skin, eyes and eye crystalline and so on. Some of these tissues have a distinguished threshold while other are displayed within the same gray values interval. In these cases special segmentation techniques and manual editing are employed to isolate single tissues from the surroundings. The process starts from a cross section where the selected tissue boundaries are well visible. A contour is manually drawn around the region of interest (ROI) and projected to the next cross sections in both directions. If the contour fits well the new cross sectional area then it is projected unchanged forward to the next slice, otherwise it is adapted using manual editing and then projected ahead to the next slice. If the cross sectional area does not change much between slices it is enough to identify the contour in only a few slices and interpolate between them. This process continues until all cross sections containing the selected ROI are covered. The contour areas are then erased creating a gap between the ROI and surrounding. Finally, a new segmentation mask representing ROI is created applying a region growing procedure which creates a new mask separating the edited structure that is no longer connected to the surrounding tissues. Some tissues like the dura layer and the skin were segmented using other tools in mimics. The dura layer was segmented using wrapping functions on the CSF tissue mask. Similarly the skin was segmented using a wrapping function on the soft tissue mask. The results of the segmentation process are shown in Fig.1.

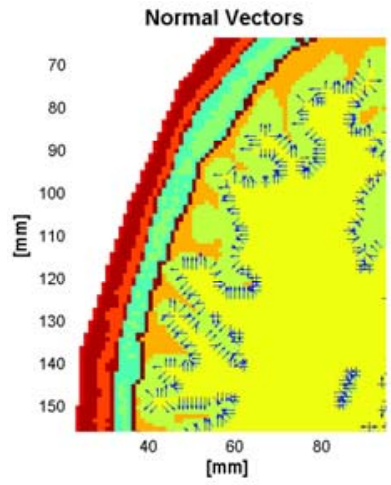

Fig 2 The Normal Vectors within a Brain Segmented Slice

\subsection{Normal vectors}

After the head has been adequately segmented the next step is to locate the surface boundary between the white and gray matter. This was done by using isosurface algorithms in Matlab version 7.10 (Mathworks Inc, USA) with a binary image matrix with one as the white matter and zero elsewhere.

After the surface nodes where extracted, normal vectors were computed using the central finite difference approximation to the numerical gradient at those nodes in the binary matrix. So for each surface node $f(x, y, z)$ the three gradient components are found by:

$$
\begin{aligned}
& \frac{\partial f}{\partial x z}=f\left(x+\frac{h_{x}}{2}\right)-f\left(x-\frac{h_{x}}{2}\right) \\
& \frac{\partial f}{\partial y}=f\left(y+\frac{h_{y}}{2}\right)-f\left(y-\frac{h_{y}}{2}\right) \\
& \frac{\partial f}{\partial z}=f\left(z+\frac{h_{z}}{2}\right)-f\left(z-\frac{h_{z}}{2}\right)
\end{aligned}
$$

Where hx, hy and hz are the separation between the adjacent points in $\mathrm{x}, \mathrm{y}$ and $\mathrm{z}$ directions, respectively.

Since the surface nodes represent a level set the gradient components are perpendicular to the surface and are therefore the normal vector components. This can be seen in Fig 2 .

\subsection{Electrical activity modelling}

An example of modelling the spontaneous electrical activity of the normal brain is presented here using the normal vectors to represent location and orientation of dipoles. A finite element method (FEM) model was constructed out of 192 segmented axial slices extending from top of the head to the bottom of the neck. The voxel resolution was $1 \times 1 \times 1 \mathrm{~mm}$. The electrical conductivities of various tissues were obtained from the literature and are summarized in our previous work [10]. The conductivity of the dura matter is not well established and it was found to have a large range from 0.02 to $0.1 \mathrm{~S} / \mathrm{m}$ [8]. For our work, 


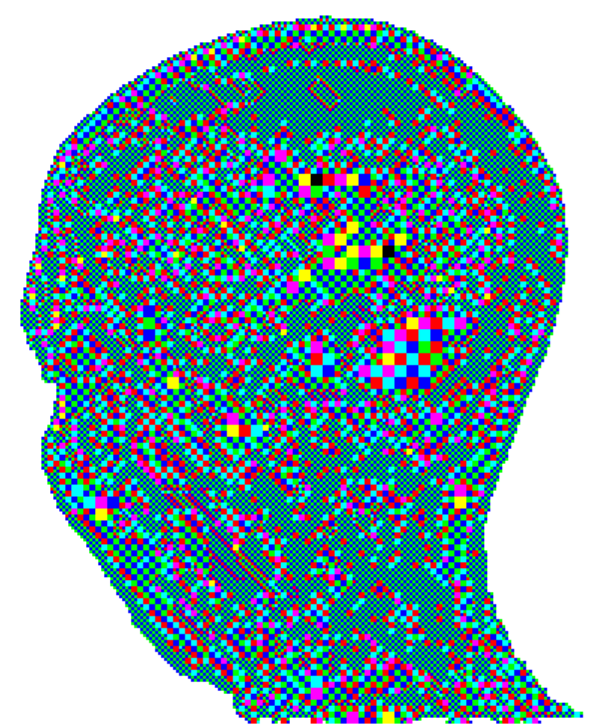

Fig $32 D$ view of the FEM mesh of the adaptive solver

we used a midrange value of $0.06 \mathrm{~S} / \mathrm{m}$. The electrical activity in the top portion of the brain, above the eye level, was simulated with 125 dipoles randomly located in different parts of the brain. The dipole intensity distribution was in the range of 0.0 to $0.4 \mathrm{~mA}$ meter with a uniform random distribution. An adaptive FEM solver, developed by us $[10,11]$, was used to compute flux and potential distribution in the whole head model. An example of an adaptive mesh is shown in Fig. 3. It shows an adaptive FEM grid and the details of the grid in the vicinity of one of the dipolar source. The FEM software automatically adjusts the grid resolution in each pass to achieve the desired L2 norm while keeping the computational errors to a minimum level. An example of the current distribution is given in Figs. 4. The current flow pattern follows the anatomical tissue boundaries very accurately. This is very pronounced at the CSF, gray and white matter boundaries. This shows that our segmentation and FEM modelling works well for computing flux and potential distributions in human head models.

\subsection{Differential contributions of dipolar activity to scalp potentials}

The above described tools were used to analyze how neuronal activity at different depths contribute towards scalp EEGs. For this purpose we computed the scalp potentials due to dipoles in two layers at two different depths. The first layer, called Layer 1, was from top of the brain surface to the depth of $1 \mathrm{~cm}$ and the second layer, called Layer 2, was from the depth of 1 to $2 \mathrm{~cm}$ from top of the brain surface. There were 2386 dipoles in the first layer and 9940 dipoles in the second layer. The total number of dipoles was 12326. The dipole intensity distribution was in the range of 0.0 to $0.4 \mathrm{~mA}$ meter with a uniform random distribution.
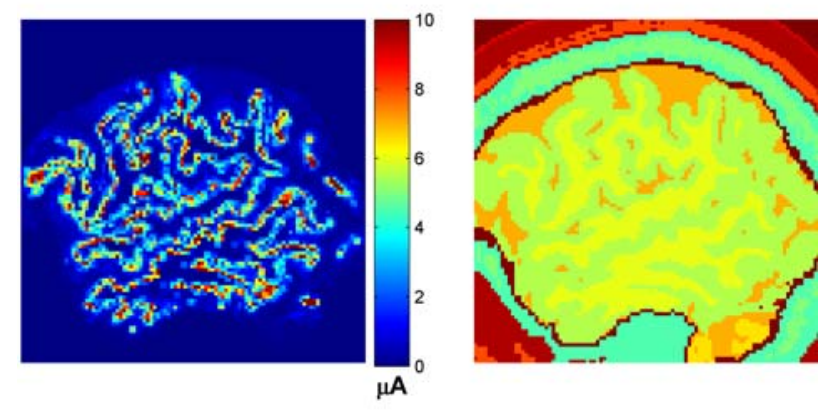

Fig 4 Current density distribution within a segmented slice (left) and the segmented slice (right). The intensity scale is in $\mu \mathrm{A} / \mathrm{cm}^{3}$

The adaptive FEM solver described above was used to compute flux and potential distribution in the whole head model. Two models were studied. For one model the dipoles in the first layer were used and in the other model the dipoles in the second layer were used. The scalp potentials were extracted from the node potentials in the finite element models of the head. All computations were performed on an Intel quad core, second generation, $2.4 \mathrm{GHz}$ workstation with $8 \mathrm{~GB}$ memory. Each run took about 10 minutes. Postprocessing and visualizations were done using Matlab software, version 7.10.

\section{Results and Discussion}

The Scalp potentials due to the first and second layers are given in Fig. 5 and a combination of both layers are given in Fig. 6. In both of these figures, the nose is on the top. The magnitude scale of the colour bar is in micro volts $(\mu \mathrm{V})$. The scalp potentials of Layer 1 and Layer 2 both have an equivalent dipolar activity patterns. For the Layer 1, the positive contours are in the middle of the scalp slightly to the right side. An equivalent dipolar source can visualized as extending from the centre of the negative contours to the centre of the positive contours. For the Layer 2, almost circular positive contours are visible in the plot. This will suggest that the equivalent dipolar activity is pointing from the bottom to the top of the head, i.e., from inferior to superior position. The combined
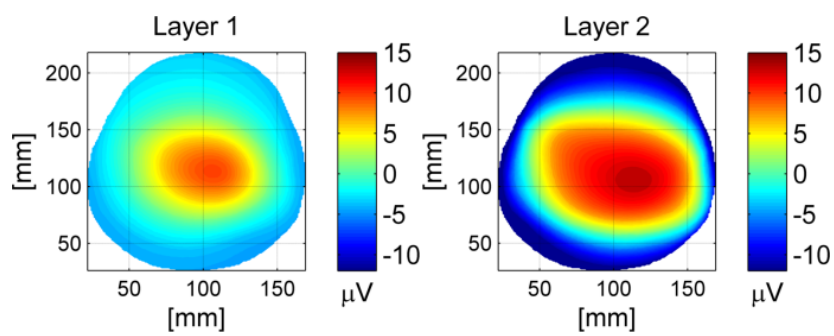

Fig 5 Scalp potentials due to dipoles in layers 1 (left) and layer 2 (right) 
3D segmented model of head

European Journal Translational Myology - Basic Applied Myology 2012; 22 (1\&2): 57-60

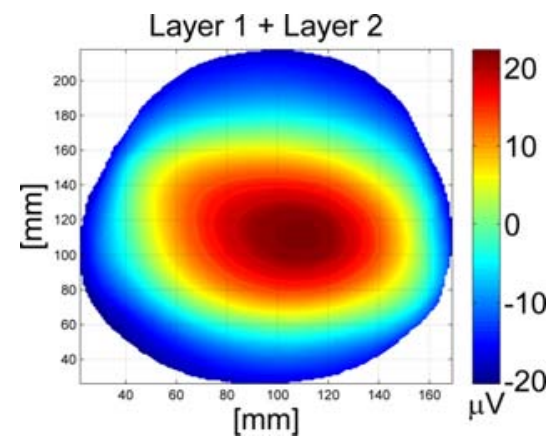

Fig 6 Scalp potentials due to layers 2.

activity of the dipolar sources in Layer 1 and Layer 2 is given in Fig. 6 and it shows that it is dominated by the scalp potentials due to dipoles in Layer 2. This feasible because there are 9940 dipoles in Layer 2 as compared with only 2386 dipoles in Layer 1.

These are our preliminary results to show our capabilities to segment the tissue boundaries with use of MIMICS software, to build detailed accurate anatomical models and to accurately compute current and potential distributions in the model. An application of our tools for differential contributions of the dipolar activity to the scalp potentials is also presented. In future, we plan to use these models for modelling of the electrical activity of the brain under normal and pathological conditions and to research the influence of different brain structures on the EEG signal

\section{Acknowledgements}

This work has been supported by The University Hospital research fund of Iceland Landspitali.

Egill Axfjord Fridgeirsson is a 24 year old master student at Reykjavik University Iceland in Biomedical Engineering. $\mathrm{He}$ is on his second year of graduate studies and this work represents part of his master thesis. His research interests are Medical modeling, Biomagnetism and clinical applications of medical models. He currently works in the Clinical Engineering department of Landspitali University hospital.

\section{Corresponding Author}

Paolo Gargiulo, Department of Development CE and IT, Landspitali University Hospital and Department of Biomedical Engineering, University of Reykjavik, Iceland E-mail: paologar@landspitali.is

Co-authors E-mails:

$(1,2)$ egilla@1sh.is;

$(2,3)$ ceonramon@yahoo.com;

(4) jens.haueisen@tu-ilmenau.de

\section{References}

[1] Abraham K. and Marsan C.A., 1958. Patterns of cortical discharges and their relation to routine scalp electroencephalography, Electroenceph. clin. Neurophysiol 10(3):447-461.

[2] Gargiulo, P., Kern, H., Carraro, U., Ingvarsson, P., Knútsdóttir, S., Guðmundsdóttir, V., Yngvason, S., Vatnsdal, B. and Helgason, T., 2010. Quantitative color 3-dimensional computer tomography imaging of human long-term denervated Muscle. Neur Res 32:13-20

[3] Gargiulo, P., Vatnsdal, B., Ingvarsson, P., Knútsdóttir, S., Gudmundsdottir, V., Yngvason, S. and Helgason, T., 2008. Restoration of Muscle Volume and Shape Induced by Electrical Stimulation of Denervated Degenerated Muscles: Qualitative and Quantitative Measurement of Changes in Rectus Femoris Using Computer Tomography and Image Segmentation. Artif Org 32: 609-613.

[4] Hallez, H., Vanrumste, B., Grech, R., Muscat, J., De Clercg, W., Verguilt, A., D'Asseler, Y., Camilleri, K.P., Fabri, S.G., Van Huffel, S. and Lemahieu, I. 2007. Review on solving the forward problem in EEG source analysis. JNER 4: 46.

[5] Linás, R.R., Nicholson, C., 1974. Analysis of field potentials in the central nervous system. Handbook Electroenceph. clin. Neurophysiol 2B:61-83.

[6] MATLAB version 7.10.0. Natick, Massachusetts: The MathWorks Inc., 2010

[7] Okada, Y.C., 1982. Neurogenesis of evoked magnetic fields. In: Williamson, S.J., Romani, G.L., Kaufman, L. \& Modena, L., eds. Biomagnetism; an Interdisciplinary Approach. New York:Plenum Press, 399-408,

[8] Oozeer M., Veraart, C., Legat, V., Delbeke, J., 2005. Simulation of intra-orbital optic nerve electrical stimulation. Med and Biolog Eng Comp 43(5):608-617.

[9] Ramon, C., Schimpf, P.H., Haueisen, J., Holmes, M, and Ishimaru, A., 2004, Role of soft bone, CSF and gray matter in EEG simulations. $B M E$ $16(4): 245-8$

[10] Ramon. C., Schimpf, P.H and Haueisen, J., 2006. Influence of head models on EEG simulations and inverse source localizations. BME 5:10

[11] Schimpf PH, Haueisen J, Ramon C, and Nowak H., 1998. Realistic computer modeling of electric and magnetic fields of human head and torso. Parallel Computing 24:1433-1460. 